\title{
Axisymmetric scattering of a fluid spheroid illuminated by an acoustical Bessel beam
}

\author{
Mingsheng Wang', Wei Li ${ }^{2}$ \\ ${ }^{1}$ Huazhong University of Science and Technology, Wuhan, China \\ ${ }^{2}$ Collaborative Innovation Center for Advanced Ship and Deep-Sea Exploration (CISSE), Shanghai, China \\ ${ }^{2}$ Corresponding author \\ E-mail: ${ }^{1}$ mingshengwang@hust.edu.cn, ${ }^{2}$ hustliw@hust.edu.cn
}

Received 4 November 2017; accepted 12 November 2017

DOI https://doi.org/10.21595/vp.2017.19383

Check for updates

\begin{abstract}
Depending on the partial wave series expression, the scattering of a fluid spheroid illuminated by a zeroth-order Bessel beam is calculated in the spherical coordinate. For a fluid spheroid, the scattering is associated with the host medium, and the immersed spheroid medium. Although spherical and cylinder fluid objects has been discussed a lot before, a prolate and oblate fluid spheroid has not been investigated deeply. In the paper, two limited boundary conditions (Neumann and Dirichlet) are presented and discussed about far-field scattering form functions. By comparing these two limited conditions, the analysis could be applied into underwater detection and acoustic tweezers, or other fields. For other fluid materials, this method could also be utilized to calculate and analyze.
\end{abstract}

Keywords: bessel beam, fluid spheroid, acoustic scattering.

\section{Introduction}

Over the past decades, there has been an amount of discussions about Bessel beams for optics [1], electromagnetic [2] and acoustical scattering [3]. When the incident plane wave encounters an obstacle, the diffraction, which could change the amplitude and phase of the sound wave, would occur. Taking the advantage of no-diffraction, the Bessel beam provides an attractive alternative to using the plane wave and Gaussian beams in a wealth number of applications. Furthermore, Bessel beams are self-healing; if the part of the beam is obstructed or distorted, the Bessel beam could recover after a characteristic propagation distance by itself.

Motivated by the prominent advantages of the Bessel beam, the acoustical scattering of the Bessel beam centered on the prolate and oblate spheroids could be worth investigating. Meanwhile, the incident acoustical field would be affected by the target object illuminated by the incident beam, since the incident wave would reflect, scatter, and refract off the targets' surface. Note that because of the geometry of the prolate and oblate spheroid different from a sphere, the analytic formula would clearly lead to the inaccuracies in the prediction of the scattering and other mechanical effects of the acoustical waves.

The rigid spheroid has been investigated for a long history. However, less attention of the liquid spheroid has been gotten till 1964 when the exact solution of the diffraction of plane sound waves by an acoustically penetrable oblate spheroid was obtained for the first time [4]. And a series of reports on the scattering of waves by a penetrable prolate spheroid and the exact solution of the scattering of acoustical waves by a liquid prolate spheroid were obtained [5]. Recently, there are more papers about the approximate solution based on the discussions before [6]. Otherwise, two different methods for a plane acoustic wave from a penetrable prolate or oblate spheroid are used for the evaluation [7]. In addition, Edmundo Lavia et al. derived a computational method to calculate the exact solution for liquid spheroid which agrees with reported predicted results obtained through approximated solution for far-field and near-field regimes [8]. Based on the discussions of the scattering of the fluid spheroid, the acoustical radiation force of a fluid spheroid or a liquid-filled spherical shell could be obtained, which could benefit the application of the biomedical, drug deliver, biochemistry and other areas [9].

For a sphere, the solution approach could be obtained by the separated variable, based on an 
expansion of the spherical wave function. Therefore, the calculation of the prolate and oblate spheroid could be gotten through this similar way.

\section{Theory}

Consider a fluid spheroid immersing in the non-viscous fluid under the illumination of an acoustical monochromatic zeroth-order Bessel beam, and the incident upon a spheroid centered on its axis of wave propagation [end-on incident (Fig. 1)]. The ideal fluid surrounding the spheroid has an acoustical velocity $c_{0}$ and a density $\rho_{0}$. The incident pressure field could be expressed as a PWSE as $[10,11]$ :

$P_{i}(r, \theta)=P_{0} \sum_{n=0}^{\infty} i^{n}(2 n+1) j_{n}\left(k_{0} r\right) P_{n}(\cos \theta) P_{n}(\cos \beta)$,

where $P_{0}$ is the acoustical pressure amplitude, $j_{n}$ is the spherical Bessel function of zeroth-order of the first kind, $P_{n}$ is the Legendre function, $k_{0}$ is the wave number of the surrounding fluid, and $\beta$ is the half-cone angle of the Bessel beam.

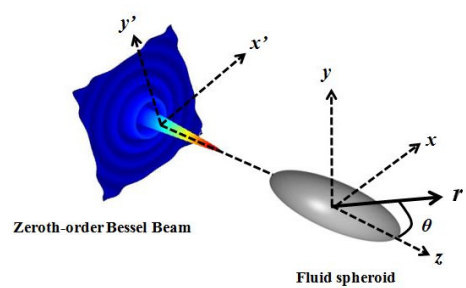

Fig. 1. A fluid spheroid illuminated by the zeroth-order Bessel beam along axis direction $z$

The complex scattered pressure field from the fluid spheroid in the surrounding non-viscous fluid could be expressed as:

$P_{S}(r, \theta)=P_{0} \sum_{n=0}^{\infty} i^{n}(2 n+1) A_{n} h_{n}^{(1)}\left(k_{0} r\right) P_{n}(\cos \theta) P_{n}(\cos \beta)$,

where $A_{n}$ is the scattering coefficient for the host medium, and $h_{n}^{(1)}$ is the spherical Hankel function of the first kind.

Since the scattering object is composed of a fluid material, the internal pressure field inside the fluid spheroid could be expressed as:

$P_{\text {int }}(r, \theta)=P_{0} \sum_{n=0}^{\infty} i^{n}(2 n+1) B_{n} j_{n}\left(k_{1} r\right) P_{n}(\cos \theta) P_{n}(\cos \beta)$,

where $B_{n}$ is the inward wave coefficient of the internal wave, $k_{1}$ is the internal wavenumber, $\rho_{1}$ is the internal density.

Since the surface shape function $S(\theta)$ of the fluid spheroid is an azimuthal symmetry rotation structure and only relies on the polar angle $\theta$, its expression could be written by:

$S(\theta)=\left(\frac{\cos ^{2} \theta}{a^{2}}+\frac{\sin ^{2} \theta}{b^{2}}\right)^{-1 / 2}$,

where parameter $a$ and $b$ are the semi-axes, respectively. 
The key point of this paper, is to apply continuity of the pressure and the normal component of the fluid media particle velocity at the boundary, $r=S(\theta)$, as:

$\left.\left(P_{i}+P_{S}\right)\right|_{r=S(\theta)}=\left.P_{\text {int }}\right|_{r=S(\theta)}$,

$\left.\frac{1}{i \rho_{0} \omega} \nabla\left(P_{i}+P_{S}\right) \cdot n\right|_{r=S(\theta)}=\left.\frac{1}{i \rho_{1} \omega} \nabla P_{i n t} \cdot n\right|_{r=S(\theta)}$,

where:

$n=e_{r}-\left(\frac{1}{S(\theta)}\right) \frac{d S(\theta)}{d \theta} e_{\theta}$

with $e_{r}$ and $e_{\theta}$ denoting the outward unit vectors along the radial and polar directions, respectively.

The coefficient $A_{n}$ and $B_{n}$ included in Eq. (2) and (3) for the fluid spheroid can be obtained after substituting Eqs. (1), (2) and (3) into Eqs. (5) and (6) through Eq. (7). This procedure leads to a system of linear equations:

$$
\begin{aligned}
& \sum_{n=0}^{\infty} i^{n}(2 n+1) P_{n}(\cos \beta)\left[\Psi_{n}^{p s}(\theta)+A_{n} \Psi_{n}^{p s}(\theta)\right]=\sum_{n=0}^{\infty} i^{n}(2 n+1) P_{n}(\cos \beta)\left[B_{n} \Psi_{n}^{p i n t}(\theta)\right], \\
& \frac{1}{i \rho_{0} \omega} \sum_{n=0}^{\infty} i^{n}(2 n+1) P_{n}(\cos \beta)\left[\Gamma_{n}^{v i}(\theta)+A_{n} \Gamma_{n}^{v s}(\theta)\right] \\
& =\frac{1}{i \rho_{1} \omega} \sum_{n=0}^{\infty} i^{n}(2 n+1) P_{n}(\cos \beta)\left[B_{n} \Gamma_{n}^{v i n t}(\theta)\right],
\end{aligned}
$$

where $\Psi_{n}^{p i}(\theta), \Psi_{n}^{p s}(\theta)$ and $\Psi_{n}^{p i n t}(\theta)$ could be given, respectively, by:

$$
\left\{\begin{array}{c}
\Psi_{n}^{p i}(\theta) \\
\Psi_{n}^{p S}(\theta) \\
\Psi_{n}^{\text {pint }}(\theta)
\end{array}\right\}=\left\{\begin{array}{c}
j_{n}\left(k_{0} S(\theta)\right) \\
h_{n}^{(1)}\left(k_{0} S(\theta)\right) \\
j_{n}\left(k_{1} S(\theta)\right)
\end{array}\right\} P_{n}(\cos \theta)
$$

where $\Gamma_{n}^{v i}(\theta), \Gamma_{n}^{v s}(\theta)$ and $\Gamma_{n}^{v i n t}(\theta)$ could be given, respectively, by:

$$
\left\{\begin{array}{c}
\Gamma_{n}^{v i}(\theta) \\
\Gamma_{n}^{v S}(\theta) \\
\Gamma_{n}^{v i n t}(\theta)
\end{array}\right\}=\left\{\begin{array}{c}
k_{0} j_{n}^{\prime}\left(k_{0} S(\theta)\right) \\
k_{0} h_{n}^{(1) \prime}\left(k_{0} S(\theta)\right) \\
k_{1} j_{n}^{\prime}\left(k_{1} S(\theta)\right)
\end{array}\right\} P_{n}(\cos \theta)-\left\{\begin{array}{c}
j_{n}\left(k_{0} S(\theta)\right) \\
h_{n}^{(1)}\left(k_{0} S(\theta)\right) \\
j_{n}\left(k_{1} S(\theta)\right)
\end{array}\right\} \frac{1}{S(\theta)^{2}} \frac{d S(\theta)}{d \theta} \frac{d P_{n}(\cos \theta)}{d \theta}
$$

To get rid of the influence, the variable angle $\theta$ in the linear Eqs. (10) and (11), PWSEs with separable variables in Eqs. (5) and (6) could be considered. Hence, Eqs. (10) and (11) could be applied into the orthogonality condition of the Legendre functions as:

$$
\frac{(2 n+1)}{2} \int_{0}^{\pi} P_{n}(\cos \theta) P_{n^{\prime}}(\cos \theta) \sin \theta d \theta=\delta_{n, n^{\prime}},
$$

where $\delta_{n, n}$, is the Kronecker delta function.

Therefore, Eqs. (8) and (9) could be rewritten by a new system of linear equations as, respectively: 
$\sum_{n^{\prime}=0}^{\infty}\left[M_{n^{\prime}}^{p i}+A_{n} M_{n^{\prime}}^{p s}\right]=\sum_{n^{\prime}=0}^{\infty}\left[B_{n} M_{n^{\prime}}^{p i n t}\right]$,
$\frac{1}{i \rho_{0} \omega} \sum_{n^{\prime}=0}^{\infty}\left[N_{n^{\prime}}^{v i}+A_{n} N_{n^{\prime}}^{v s}\right]=\frac{1}{i \rho_{1} \omega} \sum_{n^{\prime}=0}^{\infty}\left[B_{n} N_{n^{\prime}}^{v i n t}\right]$,

where:

$$
\begin{aligned}
& \left\{\begin{array}{c}
M_{n \prime}^{p i} \\
M_{n \prime}^{p s} \\
M_{n \prime}^{p i n t}
\end{array}\right\}=\sum_{n=0}^{\infty} i^{n}(2 n+1) P_{n}(\cos \beta) \int_{0}^{\pi}\left\{\begin{array}{c}
\Psi_{n}^{p i}(\theta) \\
\Psi_{n}^{p s}(\theta) \\
\Psi_{n}^{p i n t}(\theta)
\end{array}\right\} P_{n^{\prime}}(\cos \theta) \sin \theta d \theta, \\
& \left\{\begin{array}{c}
N_{n^{\prime}}^{v i} \\
N_{n^{\prime}}^{v s} \\
N_{n^{\prime}}^{\text {vint }}
\end{array}\right\}=\sum_{n=0}^{\infty} i^{n}(2 n+1) P_{n}(\cos \beta) \int_{0}^{\pi}\left\{\begin{array}{c}
\Gamma_{n}^{v i}(\theta) \\
\Gamma_{n}^{v s}(\theta) \\
\Gamma_{n}^{v i n t}(\theta)
\end{array}\right\} P_{n^{\prime}}(\cos \theta) \sin \theta d \theta .
\end{aligned}
$$

The far field form function could be expressed as:

$f_{\infty}\left(k r_{0}, \theta, \beta\right)=\frac{2}{i k r_{0}} \sum_{n=0}^{\infty}(2 n+1) A_{n} P_{n}(\cos \beta) P_{n}(\cos \theta)$,

where $r_{0}=\max (a, b)$.

\section{Numerical results}

Note that a water spheroid in air, the water drop could be defined as a rigid spheroid, since there is large mismatch impendence between liquid and air, which could be considered as the Neumann boundary condition, meaning the normal velocity between the fluid prolate and oblate spheroid and the host medium is zero. Based on this character, the correction of the fluid spheroid could be verified. Meanwhile, for a gas-filled spheroid in water, which is different from a water spheroid, the boundary condition could be considered as Dirichlet, meaning the pressure between the fluid prolate and oblate spheroid and the host medium is zero. Then, we will discuss about these two conditions above. The analysis will center on backscattering form function, polar scattering form function, and near-field scattering pressure. Because of the accuracy of the spherical Hankel functions with the increasing number of terms based on PWSE in this paper, the calculation method could only be applied into calculation and analysis for a prolate and oblate spheroid with a maximum aspect ratio of $3 / 1$ or a minimum aspect ratio of $1 / 3$.

Note that when $\beta=0^{\circ}$, the incident wave corresponds to the plane wave. Consider the example that $\rho_{\text {water }}=1000 \mathrm{~kg} / \mathrm{m}^{3}, c_{\text {water }}=1480 \mathrm{~m} / \mathrm{s}, \rho_{\text {air }}=0.0014 \mathrm{~kg} / \mathrm{m}^{3}$ and $c_{\text {air }}=340 \mathrm{~m} / \mathrm{s}$. The corresponding results of the Neumann boundary influenced by the Bessel cone are shown in Fig. 2 and Fig. 3. The 3D far-field form function directivity patterns of the Neumann boundary condition are shown in Fig. 2, with $a / b=1 / 3, a / b=1 / 2, a / b=2 / 1, a / b=3 / 1$ for $k b=5$ or $k a=5$. Fig. 3 shows the $3 \mathrm{D}$ far-field form function directivity patterns of the Dirichlet boundary condition, respectively. Also, the fluid prolate and oblate spheroid is illuminated by the zeroth-order Bessel beam (half-cone angle $\beta=0^{\circ}, \beta=30^{\circ}, \beta=60^{\circ}$ ), which is correspondent to the form function for different dimensionless frequency.

By comparing the Neumann boundary condition and the Dirichlet boundary condition, there are not many differences between two conditions for the ratio $a / b=1 / 3,1 / 2$ under the half-cone Bessel beam angle $\beta=0^{\circ}$ and $\beta=30^{\circ}$. However, for $\beta=60^{\circ}$, the middle scattering directivity tends towards the back for Neumann condition, which tends forward for Dirichlet condition in contrast. As observed in Fig. 2(g)-(1) and Fig. 3(g)-(1), the scattering of the fluid spheroid in the 
forward half part is obvious for two different conditions clearly. However, the backward depression for Neumann condition would be a little stronger than that for the Dirichlet condition. By comparing with two limited conditions for the Neumann condition and the Dirichlet condition, analysis of the scattering form function would be helpful to detect the prolate and oblate objects underwater.

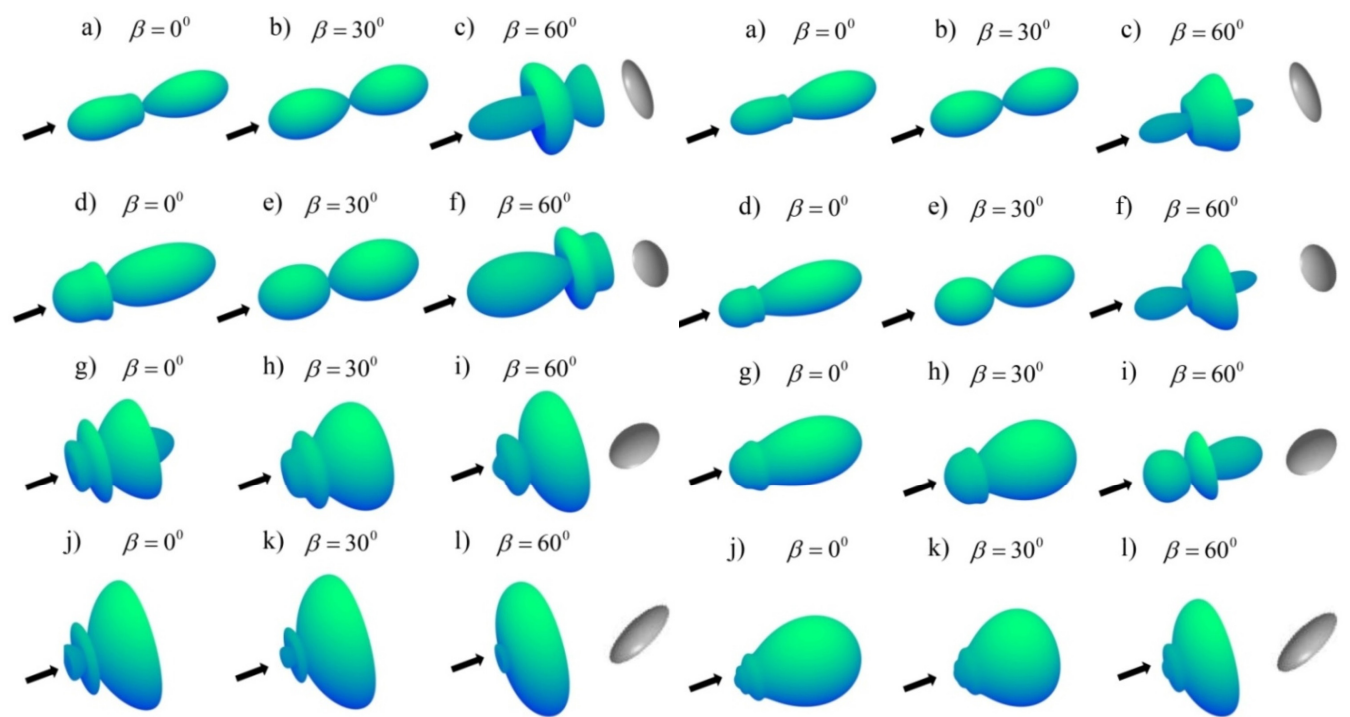

Fig. 2. The 3D directivity patterns of the Neumann boundary for Bessel half-cone angles $\beta=0^{\circ}, 30^{\circ}$ and $60^{\circ}$ at $k r_{0}=5:$ a)-c) $a / b=1 / 3$, d)-f) $a / b=1 / 2$, g)-i) $a / b=2 / 1, \mathrm{j})-1) a / b=3 / 1$
Fig. 3. The 3D directivity pattern sof the Dirichlet boundary for Bessel half-cone angles $\beta=0^{\circ}$, $30^{\circ}$ and $60^{\circ}$ at $k r_{0}=5:$ a) -c) $a / b=1 / 3$, d)-f) $a / b=1 / 2$, g)-i) $a / b=2 / 1$, j)-1) $a / b=3 / 1$

\section{Conclusions}

Through the discussions about the fluid spheroid illuminated by a zeroth-order Bessel beam along the axial incidence direction under two limited boundary condition, the form functions about a prolate and oblate fluid spheroid could be obtained based on the separation of variables in spherical coordinate. It is confirmed that the spherical coordinate could be applied into solution of a prolate and oblate spheroid in some degree, not only spherical fluid particles. Meanwhile, the results for the Neumann boundary condition has been obtained, hence the consequence of the Dirichlet boundary condition could also been gotten easily. Additionally, the pressure field for the incident wave and the scattering wave could be derived, and the nature could be obtained easily with particular emphasis on the half-cone angle of the Bessel beam, the dimensionless frequency, and the ratio of the spheroid. By comparing two boundary conditions, the properties of the scattering for a fluid spheroid could be distinguished easily, thus these could also be applied into practical detection underwater.

\section{References}

[1] Turunen J., Vasara A., Friberg A. T. Holographic generation of diffraction-free beams. Applied Optics, Vol. 27, Issue 19, 1988, p. 3959-3962.

[2] Chen Z., Han Y., Cui Z. Scattering of Bessel beam by a conducting spherical particle with dielectric coating. Journal of Quantitative Spectroscopy and Radiative Transfer, Vol. 148, 2014, p. 197-202.

[3] Mitri F. G. Acoustic scattering of a high-order Bessel beam by an elastic sphere. Annals of Physics, Vol. 323, Issue 11, 2008, p. 2840-2850.

[4] Yeh C. The diffraction of sound waves by penetrable disks. Annalen der Physik, Vol. 468, Issues 1-2, 1964, p. 53-61. 
[5] Yeh C. Scattering of acoustic waves by a penetrable prolate spheroid. The Journal of the Acoustical Society of America, Vol. 42, Issue 2, 1967, p. 518-521.

[6] Prario I. S., Gonzalez J. D., Madirolas A. A Prolate spheroidal approach for fish target strength estimation: modeling and measurements. Acta Acustica united with Acustica, Vol. 101, Issue 5, 2015, p. 928-940.

[7] Kotsis A. D., Roumeliotis J. A. Acoustic scattering by a penetrable spheroid. Acoustical Physics, Vol. 54, Issue 2, 2008, p. 153-167.

[8] González J. D., Lavia E. F., Blanc S. A computational method to calculate the exact solution for acoustic scattering by fluid spheroids. Acta Acustica united with Acustica, Vol. 102, Issue 6, 2016, p. 1061-1071.

[9] Yingbin Chai, Zhixiong Gong, Wei Li, Tianyun Li, Qifan Zhang, Zhihong Zou Application of smoothed finite element method to two dimensional exterior problems of acoustic radiation. International Journal of Computational Methods, 2018, https://doi.org/10.1142/S0219876218500299.

[10] Mitri F. G. Axisymmetric scattering of an acoustical Bessel beam by a rigid fixed spheroid. IEEE Transactions on Ultrasonics, Ferroelectrics, and Frequency Control, Vol. 62, Issue 10, 2015, p. $1809-1818$.

[11] Li Y., Wang M., Li W. Sound Scattering of Double Concentric Elastic Spherical Shell with Multilayered Medium Cloak. Underwater Technology, Busan, 2017. 